Short reports

\section{Flexible bronchoscopy via the laryngeal mask: a new technique}

\author{
C J McNamee, B Meyns, K M Pagliero
}

\begin{abstract}
Malignant tracheal tumours often cause airway obstruction and this may be aggravated by vocal cord paralysis due to invasion of the recurrent laryngeal nerve. Conventional endoscopic techniques performed under general anaesthesia do not give a simultaneous view of vocal cord function and the distal airways. The technique of bronchoscopy via the laryngeal mask allowed full assessment of the cause of stridor in a patient with a malignant tracheal tumour that was causing airways obstruction and vocal cord paralysis.
\end{abstract}

Flexible bronchoscopy is performed under general anaesthesia by about $12 \%$ of practitioners in the United Kingdom. ${ }^{1}$ Bronchoscopic evaluation of the tracheobronchial tree is an essential part of the assessment of suspected airway obstruction. Most tracheal tumours are malignant and most present with acute symptoms of dyspnoea that may mimic asthma. ${ }^{2}$ Malignant tracheal neoplasms may also invade the recurrent laryngeal nerve and cause vocal cord paralysis, though most cases of vocal cord paralysis from malignancy are due to invasion of the subaortic area by lymph node metastases from bronchial carcinoma. ${ }^{3}$ The combination of cord paralysis with obstruction of the tracheal lumen can cause acute stridor. We report a case where full assessment by flexible bronchoscopy under general anaesthesia was possible with the aid of a laryngeal mask (figure). This device consists of an elliptical cuff that is inflated in the hypopharyn $\mathrm{x}^{4}$ and allows an adequate airway under general anaesthesia without the need for laryngoscopy or tracheal intubation.

\section{Case report}

A 70 year old woman with a history of chronic smoking presented with stridor. She refused flexible bronchoscopy under local anaesthesia. General anesthesia was induced and maintained with propofol; spontaneous ventilation was used with the laryngeal mask, through which flexible bronchoscopy was performed. This revealed left vocal cord paralysis and a squamous cell carcinoma affecting the left side of the distal trachea. There was no prior history of recurrent laryngeal nerve damage, so nerve paralysis was attributed to carcinomatous infiltration. A tracheostomy was performed to relieve the stridor, so that urgent radiotherapy could be started.

\section{Discussion}

Flexible bronchoscopy can be performed easily via the laryngeal mask in a patient under light general anaesthesia induced by a drug such as propofol. A full examination of the larynx, trachea, and bronchial tree can be completed with this technique without the potential damage caused by endotracheal intubation. ${ }^{56}$ Furthermore, no paralysing agent is required, so that an unhurried and full examination of vocal cord function is possible; with rigid bronchoscopy the operator must wait for recovery from anaesthesia. Spontaneous ventilation is also maintained, which is particularly important with tracheal obstructions. ${ }^{7}$ If the operator is concerned about further loss of lumen patency resulting from bleeding or oedema from a biopsy he can defer this part of the procedure. A subsequent examination with a rigid bronchoscope would allow any bleeding to be dealt with more effectively. No obstruction to air flow occurs through the lumen of the airway (internal diameter $14 \mathrm{~mm}$ ) as the external 
diameter of the fibreoptic bronchoscope is only $5 \mathrm{~mm}$. We believe that this is a safe technique for flexible bronchoscopy under general anaesthesia. We do not, however, recommend it for patients likely to aspirate or for the control of massive haemoptysis.

1 Simpson FG, Arnold AG, Purvis A, Belfield PW, Muers MF, Cooke NJ. Postal survey of bronchoscopic practice by physicians in the United Kingdom. Thorax 1986;41:311-7
2 Xu LT, Sun ZF, Wu L.H, Wang ZZ. Tracheobronchial tumors: an eighteen year series from Capital Hospital, Peking, China. Ann Thorac Surg 1983;35:590-6.

3 Stell PM, Maran AGD. Head and neck surgery. London: Heinemann, 1978:194-204.

4 Brain AIJ. The laryngeal mask-a new concept in airway management. Br.J Anuesth 1983;55:801-5.

5 Kambic $\mathrm{V}$, Radzel $\mathrm{Z}$. Intubation lesions of the larynx. $\mathrm{Br} . \mathrm{J}$ Anaesth 1978;50:587-90.

6 Brain AIJ, McGhee TD, Miatier EJ, Thomas A, Abu-Saael MAW, Bushman JA. The laryngeal mask airway. Anaesthesia 1985;40:356-61.

7 Geffin B, Bland J, Grillo HC. Anaesthetic management of tracheal resection and reconstruction. Anaesth Analg 1969:48:884-90.

Iron lung:

bronchoscopic and

pathological

consequences of

aspiration of ferrous

sulphate

David J Godden, Keith M Kerr,
Stephen J Watt, Joseph S Legge

\begin{abstract}
Acute bronchial damage was caused by aspiration of a ferrous sulphate tablet, early histological changes (unlike in the few previously reported cases) being observed in the biopsy specimens.
\end{abstract}

Foreign body aspiration occurs mainly in children' but may occur in adults, particularly in the presence of oesophageal abnormalities. We describe the radiological, bronchoscopic, and histological findings in an elderly women with a hiatus hernia who aspirated a ferrous sulphate tablet.

\section{Case report}

An 84 year old woman, a non-smoker, suddenly developed vigorous coughing, which produced a cupful of blood. The cough persisted for four weeks until presentation, and right pleuritic chest pain developed. The patient had a hiatus hernia but no previous history of dysphagia, and was taking ferrous sulphate tablets, $200 \mathrm{mg}$ thrice daily, for chronic blood loss from the hiatus hernia. She could not recall a specific episode of aspiration.

A chest radiograph showed an incarcerated hiatus hernia and patchy opacification in the right mid and lower zones. Thoracic computed tomography showed concentric narrowing of the right lower lobe bronchus and patchy linear subsegmental collapse in the posteromedial aspect of the right lower lobe.

At bronchoscopy the right main and inter- mediate bronchi were lined with greenish brown necrotic material. The left main bronchus was entirely normal. The right intermediate bronchus was partially occluded by a hard polypoid mass covered with similar necrotic material. A bronchial biopsy specimen was taken, which histologically consisted of proteinaceous debris and elements of partially necrotic fibroconnective tissue. Fragments of disrupted bronchial epithelium were clearly identifiable and these, together with the subepithelial connective tissue, were encrusted with golden brown pigment that showed a very strong reaction for ferric iron (Prussian blue). Iron had precipitated particularly heavily on elastic fibres and bronchial epithelial cells, including their cilia (fig 1). Overlying some of the degenerate tissue was a reactive metaplastic squamous epithelium, devoid of iron precipitate.

Repeat bronchoscopy, seven days later, showed similar appearances but the polypoidal lesion was no longer visible. The

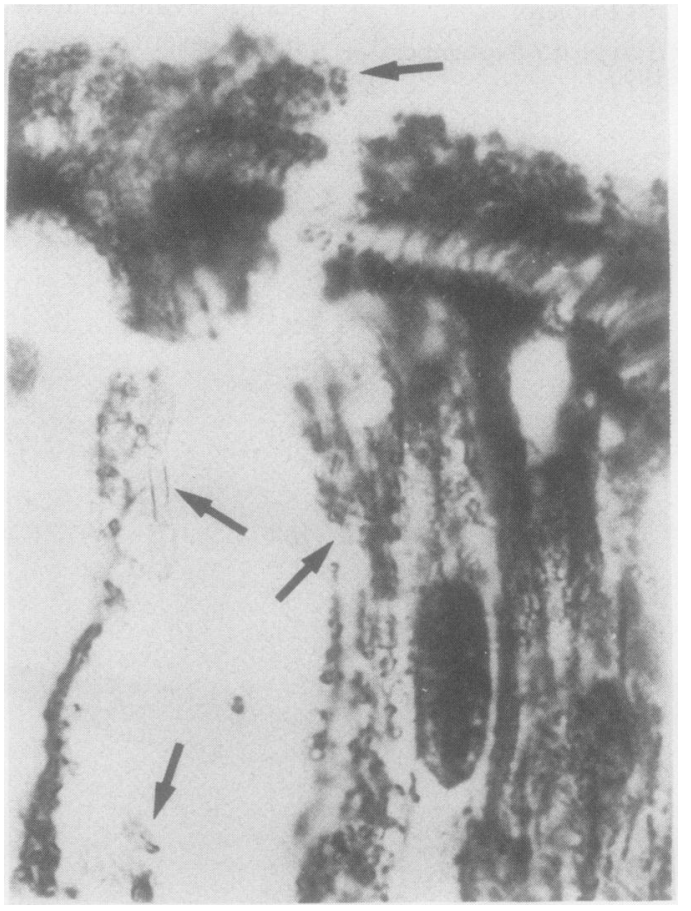

Figure 1 High power view of fragmented bronchial epithelial cells. Superiorly cilia are well seen and centrally the columnar cell nucleus is in focus. Iron salt crystals , arrows, are precipitated on cilia as well as cell membrane's and cytoplasm. (Haematoxylin and eosin.) 